\title{
Anisotropic Median-Diffusion for Filtering Noisy Electrocardiogram Signals
}

\author{
Marco A. A. de Melo, Hae Yong Kim, Denys E. C. Nicolosi
}

\begin{abstract}
Low Noise Electrocardiogram (ECG) has been widely used for heart disease diagnosis. The anisotropic median-diffusion is the filter obtained by intercalating a median filtering in each diffusion step. We propose to use anisotropic median-diffusion to filter noisy ECG signals. We describe how to estimate appropriate parameters of the proposed filter. We validate our method using ECG signals from the MIT-BIH databases (many of them with premature ventricular contraction) and compare our method with other filtering methods. Experiments show that the proposed technique can effectively remove the noise without changing the instants and amplitudes of events, as well as preserving the morphologies of ECG signals in sections of the QRS complex.
\end{abstract}

\section{INTRODUCTION}

$\mathrm{E}$ lectrocardiogram (ECG) is used to provide clinical information about heart diseases. In practice, the reliability of a diagnosis depends on the quality of the available ECG signal. An ECG acquired in a clinical environment may be corrupted by electromagnetic interferences from nearby instruments, noise from the analogdigital conversor of the ECG acquiring device, and from the muscular movements of the patient [1]. Consequently, ECG signals may be filtered to attenuate the distortions introduced by these noise sources. Linear filters are not appropriate for this task, because they usually modify the instants and amplitudes of events and change the shape of the ECG signal.

Consequently, some nonlinear filters have been proposed to filter ECG signals, as the Nonlinear Noise Reduction algorithm (NNR) [2] and Independent Component Analysis (ICA) [3]. The basic idea of NNR is to determine the underlying dynamic system to attenuate the noise. This may be achieved using local linear model. The ICA is a statistical technique for decomposing a dataset into independent subparts. NNR is better than ICA for recovering the morphology of ECG signals, and less

The authors would like to thank FEI and CNPq for the partial finantial support of this work.

M. A. A. Melo is with the Universidade de São Paulo, Escola Politécnica; and with the Centro Universitário da Fundação Educacional Inaciana (FEI), Dept. Eng. Elétrica, Av. Humberto A. de Castelo Branco, 3142, 09850-901, São Bernardo do Campo, SP, Brasil. e-mail: marco.melo@poli.usp.br.

H. Y. Kim is with the Universidade de São Paulo, Escola Politécnica, Av. Prof. Luciano Gualberto, tr. 3, 158, 05508-900, São Paulo, SP, Brazil. e-mail: hae@lps.usp.br.

D. E. C. Nicolosi is with the Instituto Dante Pazzanese de Cardiologia, Av. Dr. Dante Pazzanese, 500, 04012-909, São Paulo, SP, Brazil; and with the Centro Universitário da Fundação Educacional Inaciana (FEI). e-mail: denys@ecgweb.com.br. prone to modify the shape of ECG signals than ICA. However, ICA is better for recovering specific points of the ECG signals, like the R peak, that is used to obtain RR intervals. Wiener filter is also used to filter ECG signals, but the results are usually inferior to those obtained using NNR or ICA.

Some nonlinear filters can preserve the shapes, edges and amplitudes of the signal at the same time as filtering out the noise [4, 5]. Anisotropic diffusion is one of them. Anisotropic diffusion was originally developed within the scale space theory. Scale space is a theory that deals with the multi-resolution problem formulated by Witkin [6]. It uses Gaussian filters to obtain signals/images in coarse scales. Perona and Malik [7] proposed to substitute the Gaussian filter by the nonlinear anisotropic diffusion to generate coarse scale signals/images. Anisotropic diffusion has been successfully used in restoration, segmentation and edge detection of images/signals. Black et al. modified the anisotropic diffusion using robust statistics, obtaining the robust anisotropic diffusion [8]. Anisotropic diffusion is appropriate for filtering noises whose amplitude is lower than the "edges" of the signal, but it is not appropriate for filtering impulsive (salt and pepper) noise.

The median filter is another edge-preserving nonlinear filter. The idea is to examine a sample of the input and decide if it is representative of the signal/image. This is performed using a sliding window and computing the median of the values inside the window. Median filter is particularly useful to reduce impulsive noise.

To get the advantages of both anisotropic diffusion and median filter, some papers like [9], have suggested to intercalate median and anisotropic diffusion, to obtain a median-diffusion able to filter both Gaussian and impulsive noises.

In this paper, we analyze the possibility of filtering ECG signals using the anisotropic median-diffusion. We show that the median-diffusion can effectively remove the noise. We also show how to estimate appropriate parameters.

It is important to test the ECG filters in signals with abnormalities, because these abnormalities are important for the heart diseases diagnosis. In the present paper, we use ECG signals with premature ventricular contraction [10] from the MIT-BIH database [11].

\section{ANISOTROPIC MEDIAN-DifFUSION}

Perona and Malik [7] defined the discrete anisotropic diffusion as follows: 
$I_{s}^{t+1}=I_{s}^{t}+\frac{\lambda}{\left|\eta_{s}\right|} \sum_{p \in \eta_{s}} g\left(\nabla I_{s, p}\right) \nabla I_{s, p}, \quad i \geq 0$

where:

- $I_{s}^{t}$ is the value of the signal at iteration $t$ and point $s$.

- $\lambda \in[0,1]$ is a scalar constant that controls the diffusion rate.

- $\eta_{s}$ is the set of neighbors of the point $s$.

- $\left|\eta_{s}\right|$ is the number of neighbors of $s$, usually two for signals and four for images.

Perona and Malik approximated the derivative/gradient $\nabla I_{s, p}\left(p \in \eta_{s}\right)$ as:

$\nabla I_{s, p}=I_{p}-I_{s}$

The behavior of the anisotropic diffusion depends heavily on the choice of the "edge-stopping function" $g$. We list below three possible edge-stopping functions $g_{1}$, $g_{2}$ and $g_{3}$. The first two were proposed by Perona-Malik and the third (based on robust statistics and named "Tukey's biweight") by Black et al. [8]:

$$
\begin{aligned}
& g_{1}(x)=\frac{1}{1+\frac{x^{2}}{\sigma^{2}}} \\
& g_{2}(x)=\exp \left[\frac{-x^{2}}{2 \sigma^{2}}\right] \\
& g_{3}(x)=\left\{\begin{array}{l}
{\left[1-\frac{x^{2}}{5 \sigma^{2}}\right]^{2},\left|\frac{x^{2}}{5}\right| \leq \sigma^{2}} \\
0, \text { otherwise }
\end{array}\right.
\end{aligned}
$$

The three functions were normalized so that their "influence functions" $\psi_{i}(x)=x g_{i}(x), i=1,2,3$ have their local maxima at $x=1$. This is the point that separates noise from the useful information (also called "edges" or "outliers"). Note that the edge-stopping functions have a scale parameter $\sigma$. Black et al. suggested using the "robust scale" $\sigma_{e}$ to automatically estimate an appropriate scale:

$$
\begin{aligned}
\sigma_{e} & =1.4826 \mathrm{MAD}(\nabla I) \\
& =\operatorname{median}_{I}\left[\left\|\nabla I-\operatorname{median}_{I}(\|\nabla I\|)\right\|\right]
\end{aligned}
$$

where "MAD" denotes the median absolute deviation. For a discrete signal/image, the robust scale is computed using the derivative/gradient magnitude approximation introduced before. However, robust scale must be considered only as an "initial guess" of the really optimal scale, because the threshold between "noise" and "useful information" depends on the application and is not possible to compute it automatically.

Anisotropic diffusion is not appropriate for filtering impulsive noise. So, some papers [9] have suggested intercalating median and anisotropic diffusion, to obtain the advantages of both median and diffusion filters. To better preserve the edges, it is also possible to not apply the median filter at the edges. That is, if the local derivative/gradient is greater than the scale parameter $\sigma$, the median filter is not applied.

\section{EXPERIMENTS}

There are many questions to be answered to determine the best anisotropic diffusion for filtering ECG signals. The first is to establish which edge-stopping function is the best. The second is to find the appropriate scale $\sigma$ and the appropriate number of iterations $t_{\max }$. The third is to decide whether the simple anisotropic diffusion (a), the median-diffusion (b) or the median-diffusion where the median is not applied at the edges (c) is the best strategy.

To compare different filters, we generated an artificial ECG signal. Using an artificial signal, it is possible to compare the original, noisy and filtered signals to determine the noise reduction factor. A real ECG signal cannot be used for this task, because any real ECG signal contains noise. We generated an artificial ECG using the Physionet Databank ECG WaveGen [12], with $60 \mathrm{bpm}$, $1800 \mathrm{mVpp}, 360 \mathrm{sps}$ (figure 1). Then, we added $25 \%$ RMS Gaussian noise.

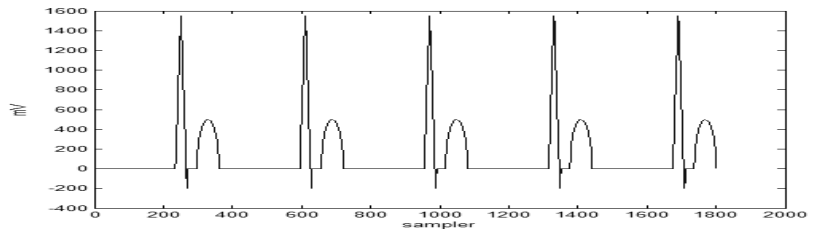

Fig.1. The artificial ECG with 1800 samples, $60 \mathrm{bpm}$, amplitude 1800 $\mathrm{mVpp}$, sampling frequency $360 \mathrm{sps}, 6$ seconds of duration.

We use the noise reduction factor as the performance metric:

$$
\delta=\sqrt{\frac{\sum_{n}[x(n)-s(n)]^{2}}{\sum_{n}[\hat{x}(n)-s(n)]^{2}}}
$$

where $s$ is the original noiseless signal, $x$ is the noisy signal and $\hat{x}$ is the filtered signal.

\section{A. Artificial ECG with Perona-Malik's function $g_{I}$}

We filtered the noisy ECG signal with the anisotropic diffusion using the robust scale $\sigma_{e}$ and the edge-stopping functions $g_{1}, g_{2}$ and $g_{3}$. The results are depicted in figure 2. It is clear that the function $g_{1}$ yields the largest noise reduction factor. We tested some other scale parameters, obtaining similar results.

Then, we found out that the largest noise reduction factor is obtained using the scale parameter about $\sigma=1.7 \times \sigma_{e}$. We tested the simple anisotropic diffusion (a), the median-diffusion (b) and the median-diffusion where the median is not applied at the edges (c) and the results were very similar. However, the method (c) yielded a slightly larger noise reduction factor. We always use median filters with window's size 3. Table I summarizes some of our tests.

The main problem of this method is to estimate the appropriate number of iterations. 
Figure 2 shows that this choice is essential, because the result deteriorates quickly before and after the optimal number of iterations. In the literature, there are some papers that try to automatically determine the diffusion stopping instant $[13,14]$, but their aptness for filtering ECG is still to be tested.

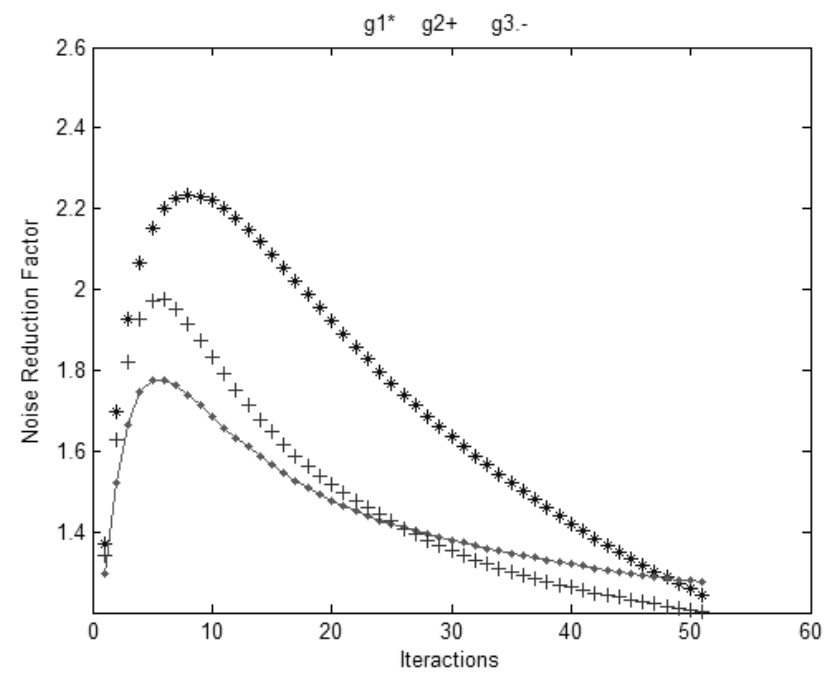

Fig.2. Noise reduction factors using the robust scale $\sigma_{e}$ and the edgestopping functions $g_{1}, g_{2}$ and $g_{3}$ versus number of iterations in artificial ECG.

TABLE I

NOISE REDUCTION FACTOR / THE BEST NUMBER OF ITERATIONS OBTAINED APPLYING the MEDian-Diffusion, STRATEgy (C), with Perona-MaliK's $g_{1}$ IN ARTIFICIAL ECG.

\begin{tabular}{|l|c|c|c|}
\hline \multicolumn{1}{|c|}{ Gaussian noise } & $0.8 \times \sigma_{e}$ & $\sigma_{e}$ & $1.7 \times \sigma_{e}$ \\
\hline $10 \%$ RMS & $1.665 / 4$ & $1.691 / 4$ & $1.729 / 3$ \\
\hline $25 \%$ RMS & $2.158 / 8$ & $2.234 / 8$ & $2.295 / 7$ \\
\hline $50 \%$ RMS & $2.785 / 16$ & $2.805 / 14$ & $2.772 / 12$ \\
\hline
\end{tabular}

\section{B. Artificial ECG with Tukey's function $g_{3}$}

The diffusion stopping criteria is not essential if we use Tukey's edge-stopping function $g_{3}$ instead of PeronaMalik's $g_{1}$. Using Tukey's function, in theory, the diffusion process converges after some number of iterations [9]. If this statement is true, why did not converge the diffusion using $g_{3}$ in figure 2? Because a too large parameter $\sigma$ was used. After some tests, we found out that the best scale that makes the diffusion process converge and yields high noise reduction factor is about $\sigma=0.5 \times \sigma_{e}$ (Table II). We also discovered that, using $g_{3}$, the median-diffusion yields a significantly higher noise reduction factor than the simple anisotropic diffusion (figure 3). In this case, the largest noise reduction factor was obtained using the strategy (b). We always use median filters with window's size 3 .
TABLE II

NOISE REDUCTION FACTOR / THE BEST NUMBER OF ITERATIONS OBTAINED APPLYING THE MEDIAN-DIFFUSION (B) WITH $g_{3}$ IN ARTIFICIAL ECG

\begin{tabular}{|c|c|c|c|}
\hline Gaussian noise & $0.3 \times \sigma_{e}$ & $0.4 \times \sigma_{e}$ & $0.5 \times \sigma_{e}$ \\
\hline $10 \%$ RMS & $1.708 / 100$ & $1.627 / 16$ & $1.696 / 40$ \\
\hline $25 \%$ RMS & $1.827 / 100$ & $1.834 / 100$ & $1.875 / 40$ \\
\hline $50 \%$ RMS & $1.885 / 100$ & $2.081 / 100$ & $2.032 / 100$ \\
\hline
\end{tabular}

Figure 3 shows that the diffusion process actually stops after about 40 iterations. Consequently, there is no need to estimate precisely the appropriate number of iterations $t_{\max }$. However, the observed noise reduction factors are smaller than those obtained using function $g_{1}$.

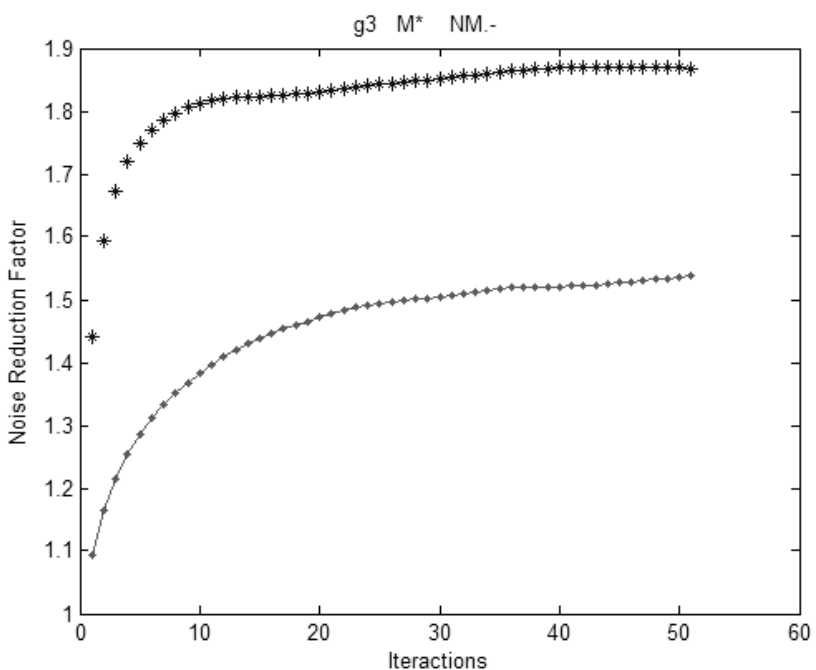

Fig.3. Upper graphic $\left(\mathrm{M}^{*}\right)$ : robust median-diffusion. Lower graphic (NM.-): robust anisotropic diffusion without median filter. In both cases, we have used Tukey's edge-stopping function $g_{3}$ and scale parameter $\sigma=0.5 \times \sigma_{e}$ in artificial ECG.

\section{Real ECG}

We used MIT-BIH database ECG samples with premature ventricular contraction to better characterize the experiments [2, 11]. We added Gaussian noise in these signals to observe the performances of the filters. Note that the noise reduction factor computed using real ECGs may not be accurate, because real ECGs always contain some quantity of noise. Figure 4 shows median-diffusion filtering process of the signal ECG_MIT-100 with premature ventricular contraction contaminated with $25 \%$ RMS Gaussian noise, using Perona-Malik's function $g_{1}$, $\sigma=1.7 \times \sigma_{e}$, and 7 iterations.

Figure 5 shows the same signal, filtered by mediandiffusion using Tukey's function $g_{3}, \sigma=0.5 \times \sigma_{e}$ and 60 iterations.

NNR nonlinear filter presents the noise reduction factor 1.99 when filtering an ECG from the MIT database contaminated with 25\% RMS Gaussian noise [2]. The Wiener filter has the noise reduction factor 1.33 in the same conditions. 
Meanwhile, the median-diffusion using PeronaMalik's $g_{1}$ has the noise reduction factor 2.003 when filtering ECG_MIT-100 signal contaminated with $25 \%$ RMS Gaussian noise. It presents the average noise reduction factor 2.0261 when applied to many ECGs with premature ventricular contractions (MIT-BIH signals 100, $102,105,114,124,205$, and 220). The median-diffusion using the Tukey's edge-stopping function $g_{3}$ presents noise reduction factor 1.839 when filtering the ECG_MIT-100 signal contaminated by $25 \%$ noise and average noise reduction factor 1.789 when applied to the same set of ECGs with premature ventricular contractions. These results demonstrate that the proposed techniques are appropriate for filtering ECG signals.
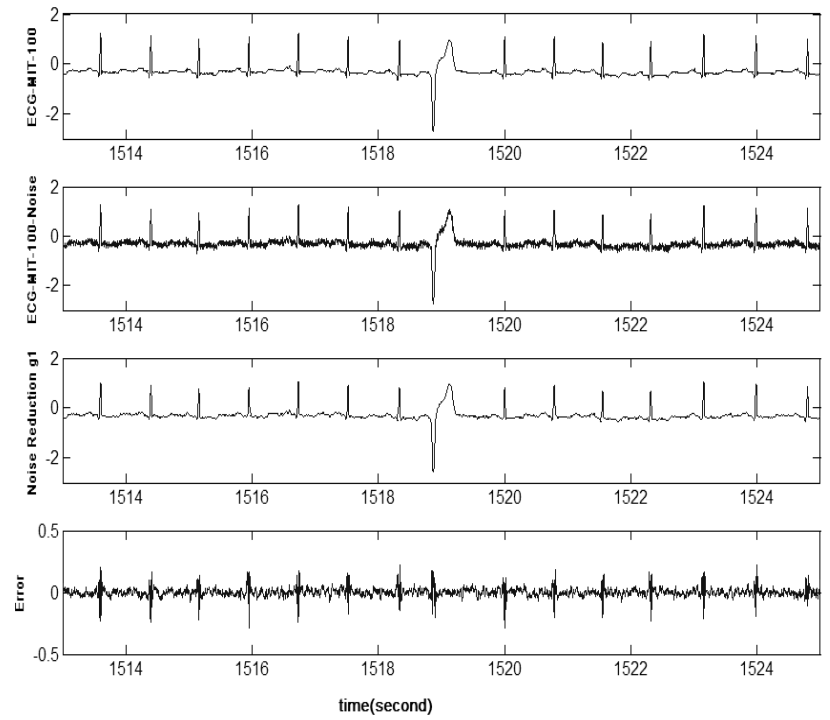

Fig.4. Top to bottom: The original ECG_MIT-100 signal. Signal contaminated with $25 \%$ RMS Gaussian noise. Signal filtered with mediandiffusion using $g_{1}, \quad \sigma=1.7 \times \sigma_{e}$, and 7 iterations. The residual error (original signal - filtered signal).
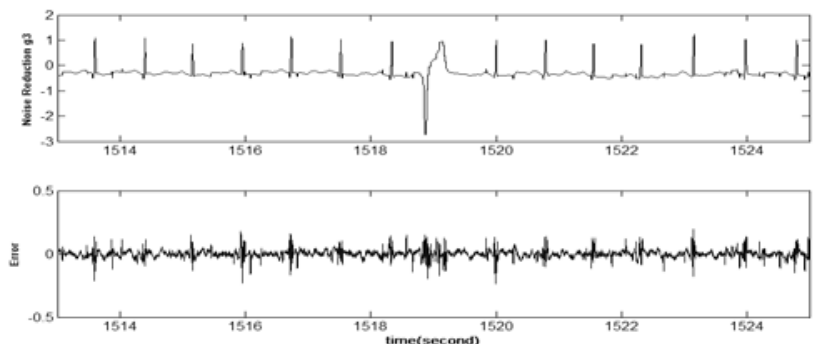

Fig.5. Top: Signal ECG_MIT-100 with $25 \%$ of noise (figure 4) filtered with median-diffusion using Tukey's $g_{3}, \sigma=0.5 \times \sigma_{e}, 60$ iterations. Bottom: The residual error (original signal - filtered signal).

\section{CONCLUSIONS}

In this paper, we have proposed to use anisotropic median-diffusion to filter noisy ECG signals. We tested three different edge-stopping functions, concluding that
Perona-Malik's function $g_{1}$ yields the largest noise reduction factor. However, in this case, it is difficult to estimate the appropriate number of iterations. Using Tukey's edgestopping function $g_{3}$, the yielded noise reduction factor is smaller. However, there is no need to estimate precisely the number of iterations, because in this case the diffusion stops after some number of iterations. The appropriate scale parameter was derived from the robust scale (that derives from the median absolute deviation). Experimental results shows that the anisotropic median-diffusion can effectively remove the noise from ECG signals without changing the details and amplitudes of the events, while preserving the morphologies of the signal in the QRS complex. In next future, we pretend to analyze the influence of the size of the median filter's window and if the proposed technique is suitable for analyzing real ECG's.

\section{REFERENCES}

[1] P. E. McSharry, G. D. Cliffordm, "Nonlinear Filtering Techniques. Advanced Methods and Tools For ECG Data Analysis," Ed. MIT. pp. 171-196, MIT 2004.

[2] T. Schreiber, D. T. Kaplan, "Nonlinear Noise Reduction for Electrocardiograms," Chaos, Vol. 6, No. 1, 1996, pp. 87-92.

[3] J. F. Cardoso, "Multidimensional Independent Component Analysis," Proc. ICASSP'98, Seattle, WA, 1998.

[4] D. D. Gobbo, M. Napolitano, P. Famouri, M. Innocenti, "Experimental Application of Extended Kalman Filtering for Sensor Validation," IEEE T. Control Systems Technology, Vol. 9, No. 2; pp. 376-380, 2001.

[5] E. Ramsden, C. Dix, "Low-Pass Filtering for Vibration Sensors," Sensor Technology and Design," Sensormag, Article 3; pp. 1-8, 2003.

[6] A. P. Witkin, "Scale Space Filtering: A New Approach to MultiScale Description," Int. Joint Conf. Artificial Intell; pp. 10191021, 1983.

[7] P. Perona, J. Malik, "Scale-Space and Edge Detection Using Anisotropic Diffusion," IEEE Trans. Pattern Anal. Machine Intell, Vol. 12; No. 7; pp. 629-634, 1990.

[8] M. J. Black, G. Sapiro, D. H. Marimont, D. Heeger, "Robust Anisotropic Diffusion," IEEE Trans. Image Processing, Vol. 7, No. 3; pp. 421-432, 1998.

[9] H. Ling, A.C. Bovik, "Smoothing low-SNR molecular images via anisotropic median-diffusion," IEEE T. Medical Imaging, vol. 21, no. 4, pp. 377-384, Apr. 2002

[10] L. C. Lantieri, J. C. Bertolett, "Interpretação Eletrocardiogáfica Adulta e Pediátrica," Artmed Ed., 2006.

[11] MIT-BIH, "Arrhythmia Database," Second-Edition, Harvard, August 1992.

[12] Physionet DataBank Available: (www.physionet.org)

[13] K. W. A. Sum ; P. Y. S. Cheung, "Stabilized Anisotropic Diffusions," Proc. ICASSP'07, Vol. 1; pp. 779-712, 2007.

[14] V. Solo, "A Fast Automatic Stopping Criterion for Anisotropic Diffusion," Proc. ICASSP'02, Vol. 2; pp. 1661-1664, 2002. 